\title{
Shape Control Learning Algorithm for Neural Networks
}

\author{
Daiyuan Zhang \\ College of Computer, Nanjing University of Posts and Telecommunications, Nanjing, 210003, P. R. China \\ E-mail: dyzhang@njupt.edu.cn, zhangdaiyuan2011@sina.com
}

\begin{abstract}
A new kind of shape control learning algorithm (SCLA) for training neural networks is proposed. We use the rational cubic spline (with quadratic denominator) to implement a new neural system for shape control, and construct a new kind of artificial neural networks based on given patterns. The shape can be controlled by some shape parameters, which is much different from the known algorithms for training neural networks. The numerical experiments indicate that the new method proposed in this paper demonstrates good results.
\end{abstract}

Keywords-neural network; weight function; rational spline; shape control

\section{INTRODUCTION}

In recent years, some new kinds of training algorithms using weight functions have been proposed to against the disadvantages in some traditional algorithms (such as BP, $\mathrm{RBF}$ algorithms). The trained weights, instead of constants, consist of weight functions defined on the sets of input variables, which has a simple architecture, good generalization and fast learning speed. In [1] and [2], the weight functions for training feedforward neural networks are cubic spline weight functions. The linear equations have to be solved for finding the cubic spline weight functions. Two different types of networks' architecture have been discussed, in which the topology is simple, the number of neurons needed for solving the problems under investigation is only dependent on the dimensions of each input and output layer. In [3], an algorithm for training neural networks based on generalized Чебышев polynomials has been proposed, in which the weight functions for training feedforward neural networks are Чебышев polynomials. Although calculation process in this algorithm has been simplified, it has reduced the level of precision. In order to find a more general expression for any order of spline functions, an algorithm of neural networks with B-spline weight functions has been proposed in [4].

In some applications, there is a need for shape control (curve or surface control), such as the outer shape of a car, a ship or an aeroplane. The methods mentioned above can hardly meet the requirement of those applications.

In this paper, we will establish the rational forms of weight functions by the given patterns, in which each of weight function is a rational function of one-variable and takes one associated input point (input neuron) as its argument. The rational cubic spline with quadratic denominator is used in this paper, and the rational cubic spline developed in this paper can be used to realize shape control.

\section{NETWORK ARCHITECTURE AND AlgORITHM}

The architecture of the neural network with weight function is showed in Fig. 1, which has only one input layer and one output layer.

Each circle in the Fig. 1 is an adder. The sign $x_{i}$ denotes the $i$-th input component of the input sample, and $z_{j}$ denotes the theoretical output value (target value) corresponding to the $j$-th neuron of output layer. Accordingly, $y_{j}$ is the real output value corresponding to the $j$-th neuron of output layer. $w_{j i}\left(x_{i}\right)$ is the theoretical weight function between the $j$-th output neuron and the $i$-th input neuron, and $R_{j i}\left(x_{i}\right)$ denotes the corresponding practical rational spline weight function.

The network's architecture is decided by the dimension of the problems. If the dimension of the input layer is $m$ and the dimension of the output layer is $n$, then there should be $m$ input nodes and $n$ output nodes, see Fig. 1 .

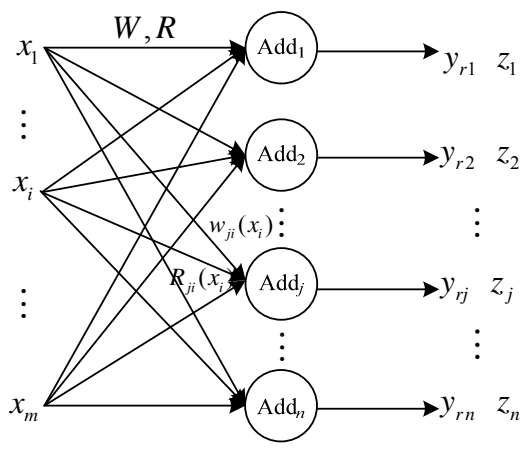

Fig. 1 The architecture of neural networks

The data of the interpolation point comes from the input and output patterns. In this paper, instead of the cubic spline weight function, the sign $R_{j i}\left(x_{i}\right)$ is the rational cubic spline with quadratic denominator, which has better performance in the condition of fractional interpolating function, extreme points and so on ${ }^{[5]}$. And there is a control parameter which can control the shape of the function curve.

Let $\left\{\left(x_{i}, f_{i}\right), i=0,1, \cdots n\right\}$ be a given set of data points, where $a=x_{0}<x_{1}<\cdots<x_{n}=b$. Let

$$
\begin{aligned}
& h_{i}=x_{i}-x_{i-1} \\
& \theta=\left(x-x_{i-1}\right) / h_{i}
\end{aligned}
$$

Then the rational cubic spline with quadratic denominator is defined as follows 


$$
R_{i}(x)=\frac{P_{i}(\theta)}{Q_{i}(\theta)}, i=1,2, \cdots n, x_{i-1} \leq x \leq x_{i}
$$

Where:

$$
\begin{gathered}
P_{i}(\theta)=(1-\theta)^{3} f_{i-1}+\theta(1-\theta)^{2}\left(r_{i} f_{i-1}+h_{i} d_{i-1}\right) \\
+\theta^{2}(1-\theta)\left(r_{i} f_{i}-h_{i} d_{i}\right)+\theta^{3} f_{i} \\
Q_{i}(\theta)=(1-\theta)^{2}+\theta(1-\theta)\left(r_{i}-1\right)+\theta^{2}
\end{gathered}
$$

The parameter $r_{i}$ is to be chosen such that $r_{i}>-1$ for a strictly positive denominator. The sign $d_{i}$ denotes the derivative values given at the knots $x_{i}$.

It is easy to calculate that $\theta\left(x_{i-1}\right)=0, \theta\left(x_{i}\right)=1$, and

$$
R_{i}\left(x_{i-1}\right)=f_{i-1}, \quad R_{i}\left(x_{i}\right)=f_{i}
$$

Calculate the derivative of (3) and (4), we have

$$
\begin{array}{r}
P_{i}^{\prime}(x)=\frac{1}{h_{i}}\left[3 \theta^{2} f_{i}+\left(r_{i} f_{i}-h_{i} d_{i}\right)\left(2 \theta-3 \theta^{2}\right)\right] \\
+\frac{1}{h_{i}}\left[\left(r_{i} f_{i-1}+h_{i} d_{i-1}\right)(1-\theta)(1-3 \theta)\right] \\
-\frac{1}{h_{i}}\left[3(1-\theta)^{2} f_{i-1}\right] \\
Q_{i}^{\prime}(x)=\frac{1}{h_{i}}\left[2\left(3-r_{i}\right) \theta+r_{i}-3\right]
\end{array}
$$

Using $\theta\left(x_{i-1}\right)=0$ and $\theta\left(x_{i}\right)=1$, we can get

$$
\left\{\begin{array} { l } 
{ P _ { i } ^ { \prime } ( x _ { i - 1 } ) = \frac { r _ { i } - 3 } { h _ { i } } f _ { i - 1 } + d _ { i - 1 } } \\
{ P _ { i } ( x _ { i - 1 } ) = f _ { i - 1 } } \\
{ Q _ { i } ^ { \prime } ( x _ { i - 1 } ) = \frac { r _ { i } - 3 } { h _ { i } } } \\
{ Q _ { i } ( x _ { i - 1 } ) = 1 }
\end{array} \left\{\begin{array}{l}
P_{i}^{\prime}\left(x_{i}\right)=\frac{3-r_{i}}{h_{i}} f_{i}+d_{i} \\
P_{i}\left(x_{i}\right)=f_{i} \\
Q_{i}^{\prime}\left(x_{i}\right)=\frac{3-r_{i}}{h_{i}} \\
Q_{i}\left(x_{i}\right)=1
\end{array}\right.\right.
$$

Since $R_{i}^{\prime}(x)=\frac{P_{i}^{\prime}(x) Q_{i}(x)-P_{i}(x) Q_{i}^{\prime}(x)}{Q^{2}(x)}$, we have

$$
R_{i}^{\prime}\left(x_{i-1}\right)=d_{i-1}, R_{i}^{\prime}\left(x_{i}\right)=d_{i}
$$

where $R_{i}^{\prime}$ denotes differentiation with respect to $x$. When $r_{i}=3$ the rational cubic clearly reduces to the standard cubic Hermite polynomials ${ }^{[6]}$. And according to (3) and (4), it is easily to get the expression of $R(x)$ if we know the derivative values and $f_{i}$.

The sign $R_{i}(x)$ is the $i$-th formula in the spline function, and the $(i+1)$-th formula is

$$
R_{i+1}(x)=\frac{P_{i+1}(x)}{Q_{i+1}(x)}
$$

$$
\begin{gathered}
P_{i+1}(x)=(1-\phi)^{3} f_{i}+\phi(1-\phi)^{2}\left(r_{i+1} f_{i}+h_{i+1} d_{i}\right) \\
+\phi^{2}(1-\phi)\left(r_{i+1} f_{i+1}-h_{i+1} d_{i+1}\right)+\phi^{3} f_{i+1} \\
Q_{i+1}(x)=(1-\phi)^{2}+\phi(1-\phi)\left(r_{i+1}-1\right)+\phi^{2}
\end{gathered}
$$

Where $\phi=\left(x-x_{i}\right) / h_{i+1}$. In the same way of the process of derivative, we can get the derivative of $R_{i+1}(x)$ where $x=x_{i}$

$$
R_{i+1}^{\prime}\left(x_{i}\right)=d_{i}
$$

and

$$
R_{i+1}^{\prime}\left(x_{i}\right)=d_{i}=R_{i}^{\prime}\left(x_{i}\right)
$$

We can see that the $R(x)$ is a continuously differentiable function.

Calculate the derivative of (6) and (7):

$$
\left\{\begin{aligned}
P_{i}^{\prime \prime}(x) & =\frac{1}{h_{i}^{2}}\left[6 \theta f_{i}+2\left(r_{i} f_{i}-h_{i} d_{i}\right)(1-3 \theta)\right] \\
& +\frac{1}{h_{i}^{2}}\left[2\left(r_{i} f_{i-1}+h_{i} d_{i-1}\right)(3 \theta-2)\right] \\
& +\frac{1}{h_{i}^{2}}\left[6(1-\theta) f_{i-1}\right] \\
Q_{i}^{\prime \prime}(x) & =\frac{1}{h_{i}^{2}}\left[2\left(3-r_{i}\right)\right]
\end{aligned}\right.
$$

Since

$$
\begin{aligned}
R_{i}^{\prime \prime}(x) & =\frac{Q_{i}^{2}(x)\left[P_{i}^{\prime \prime}(x) Q_{i}(x)-P_{i}(x) Q_{i}^{\prime \prime}(x)\right]}{Q_{i}^{4}(x)} \\
& -\frac{2 Q_{i}(x) Q_{i}^{\prime}(x)\left[P_{i}^{\prime}(x) Q_{i}(x)-P_{i}(x) Q_{i}^{\prime}(x)\right]}{Q_{i}^{4}(x)}
\end{aligned}
$$

therefore

$$
\left\{\begin{array}{l}
P_{i}^{\prime \prime}\left(x_{i}\right)=\frac{2}{h_{i}^{2}}\left[3 f_{i}-2\left(r_{i} f_{i}-h_{i} d_{i}\right)+\left(r_{i} f_{i-1}+h_{i} d_{i-1}\right)\right] \\
Q_{i}^{\prime \prime}(x)=\frac{2}{h_{i}^{2}}\left(3-r_{i}\right)
\end{array}\right.
$$

Substitute (8) and (17) into (16):

$$
R_{i}^{\prime \prime}\left(x_{i}\right)=\frac{2}{h_{i}^{2}}\left[r_{i} f_{i-1}+h_{i} d_{i-1}-r_{i} f_{i}+\left(r_{i}-1\right) h_{i} d_{i}\right]
$$

Similarly, we have

$$
\begin{aligned}
R_{i+1}^{\prime \prime}\left(x_{i}\right) & =\frac{2}{h_{i+1}^{2}}\left[2 r_{i+1} f_{i}+\left(4-r_{i}\right) h_{i+1} d_{i}\right] \\
& +\frac{2}{h_{i+1}^{2}}\left[r_{i+1} f_{i+1}-h_{i+1} d_{i+1}\right]
\end{aligned}
$$


If the control parameter $r_{i}$ satisfies

$$
\begin{aligned}
& r_{i} f_{i-1}+h_{i} d_{i-1}-r_{i} f_{i}+\left(r_{i}-1\right) h_{i} d_{i} \\
& =2 r_{i+1} f_{i}+\left(4-r_{i}\right) h_{i+1} d_{i}+r_{i+1} f_{i+1}-h_{i+1} d_{i+1}
\end{aligned}
$$

we have $R_{i}^{\prime \prime}\left(x_{i}\right)=R_{i+1}^{\prime \prime}\left(x_{i}\right)$, which means that the function $R_{i}(x)$ can be twice continuously differentiable in its domain by adjusting the parameter $r_{i}$.

Let $g(x)$ be a straight line defined in $\left[x_{i-1}, x_{i}\right]$, and

$$
Q_{i}(x)=(1-\theta)^{2}+\theta(1-\theta)\left(r_{i}-1\right)+\theta^{2} \geq 0
$$

The expression

$$
R_{i}(x)=\frac{P_{i}(x)}{Q_{i}(x)} \leq g(x)
$$

is equivalent to

$$
P_{i}(x)-Q_{i}(x) g(x) \leq 0
$$

Let $Z_{i}(x)=P_{i}(x)-Q_{i}(x) g(x)$, then

$$
\begin{aligned}
Z_{i}(x) & =(1-\theta)^{3} f_{i-1}+\theta(1-\theta)^{2}\left[r_{i} f_{i-1}+h_{i} d_{i-1}\right] \\
& +\theta^{2}(1-\theta)\left[r_{i} f_{i}-h_{i} d_{i}\right]+\theta^{3} f_{i} \\
& -\left[(1-\theta)^{2}+\theta(1-\theta)\left(r_{i}-1\right)+\theta^{2}\right] \\
& \times\left[(1-\theta) g_{i-1}+\theta g_{i}\right] \\
& =(1-\theta)^{3}\left(f_{i-1}-g_{i-1}\right)+(1-\theta)^{2} \theta E_{i} \\
& +(1-\theta) \theta^{2} F_{i}+\theta^{3}\left(f_{i}-g_{i}\right)
\end{aligned}
$$

where $g_{i}=g\left(x_{i}\right), g_{i-1}=g\left(x_{i-1}\right)$, and

$$
\left\{\begin{array}{l}
E_{i}=r_{i} f_{i-1}+h_{i} d_{i-1}-g_{i}-g_{i-1}\left(r_{i}-1\right) \\
F_{i}=r_{i} f_{i}-h_{i} d_{i}-g_{i}\left(r_{i}-1\right)-g_{i-1}
\end{array}\right.
$$

If $E_{i} \leq 0, F \leq 0$, then $Z_{i}(x) \leq 0$ is true for any $x \in\left[x_{i-1}, x_{i}\right]$. Therefore, the curve of function $R(x)$ is below the straight line $g(x)$ in $\left[x_{i-1}, x_{i}\right]$. Similarity, the curve of function $R(x)$ will be above the straight line $g^{*}(x)$ if the following holds

$$
\left\{\begin{array}{l}
r_{i} f_{i-1}+h_{i} d_{i-1}-g_{i}^{*}-g_{i-1}^{*}\left(r_{i}-1\right) \geq 0 \\
r_{i} f_{i}-h_{i} d_{i}-g_{i}^{*}\left(r_{i}-1\right)-g_{i-1}^{*} \geq 0
\end{array}\right.
$$

In other words, if the parameter $r_{i}$ satisfies the following condition

$$
\left\{\begin{array}{l}
r_{i} f_{i-1}+h_{i} d_{i-1}-g_{i}-g_{i-1}\left(r_{i}-1\right) \leq 0 \\
r_{i} f_{i}-h_{i} d_{i}-g_{i}\left(r_{i}-1\right)-g_{i-1} \leq 0 \\
r_{i} f_{i-1}+h_{i} d_{i-1}-g_{i}^{*}-g_{i-1}^{*}\left(r_{i}-1\right) \geq 0 \\
r_{i} f_{i}-h_{i} d_{i}-g_{i}^{*}\left(r_{i}-1\right)-g_{i-1}^{*} \geq 0
\end{array}\right.
$$

The cruve of function $R(x)$ will be restricted between the two straight lines of $g(x)$ and $g^{*}(x)$.

\section{Simulations}

In this simulation example, the architecture of the network is $m=1, n=1$. The target function is defined in the following

$$
y=\cos ^{2}\left(\frac{\pi x}{3}\right), 0 \leq x \leq 6
$$

$$
\begin{gathered}
g(x)= \begin{cases}1.1-\frac{2}{3} x, & 0 \leq x<1.5 \\
\frac{2}{3} x-0.9, & 1.5 \leq x<3 \\
3.1-\frac{2}{3} x, & 3 \leq x<4.5 \\
\frac{2}{3} x-2.9, & 4.5 \leq x \leq 6\end{cases} \\
g^{*}(x)= \begin{cases}0.9-\frac{2}{3} x, & 0 \leq x<1.5 \\
\frac{2}{3} x-1.1, & 1.5 \leq x<3 \\
2.9-\frac{2}{3} x, & 3 \leq x<4.5 \\
\frac{2}{3} x-3.1, & 4.5 \leq x \leq 6\end{cases}
\end{gathered}
$$

From Eq.(27), we can estimate that, when $r_{i} \geq 2$, the output values can be controlled within the two strait lines $g(x)$ and $g^{*}(x)$.

The output values of the neural network are showed in Fig. 2, Fig. 3, Fig. 4 and Fig. 5. In Fig. 2 and Fig. 3, the parameter $r_{i}<2$, some output values are beyond the two strait lines $g(x)$ and $g^{*}(x)$. In Fig. 4 and Fig. 5, the parameter $r_{i}>2$, each of output values are within the two strait lines $g(x)$ and $g^{*}(x)$.

Although the architecture of the network is $m=1, n=1$ in this example, we can generalize the network's architecture $(\mathrm{m}>1, \mathrm{n}>1)$ easily for more complicated training problems (see Fig. 1). 


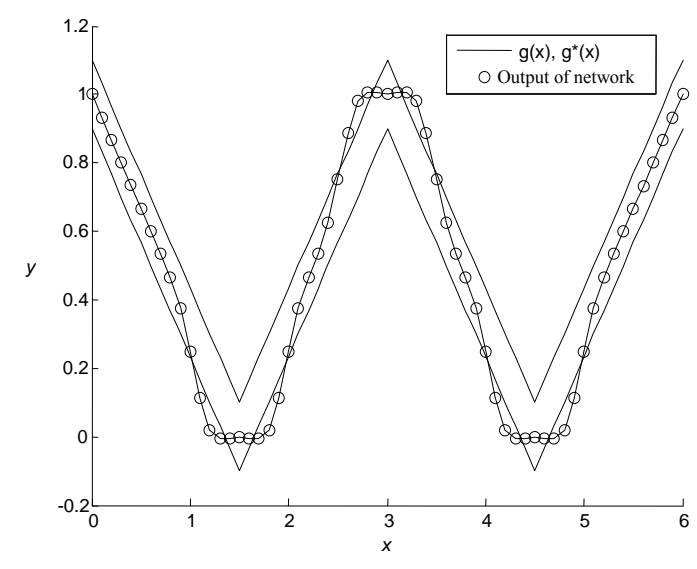

Fig. $2 r_{i}=0.5$

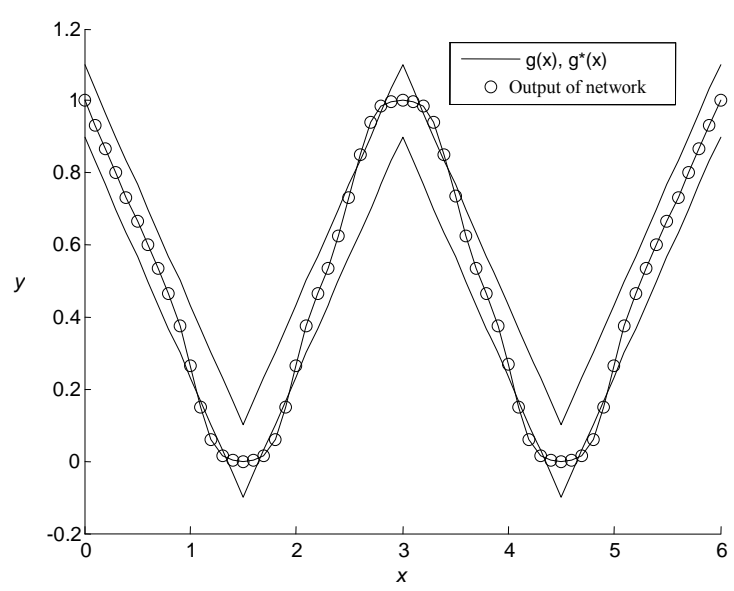

Fig. $3 r_{i}=1$

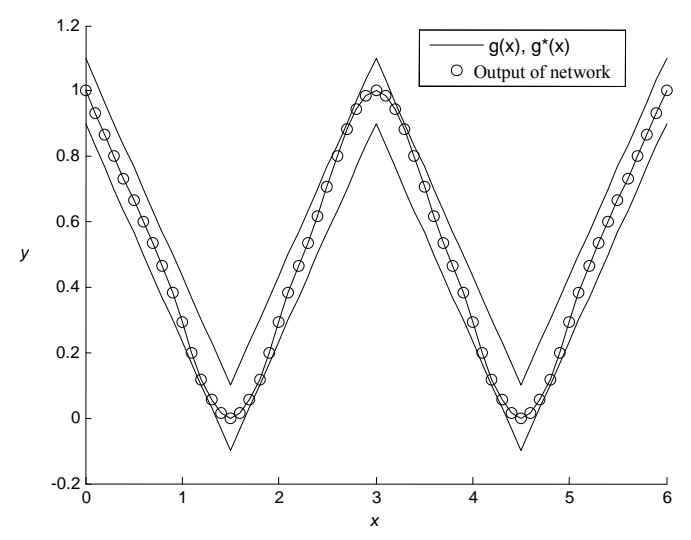

Fig. $4 r_{i}=2.5$

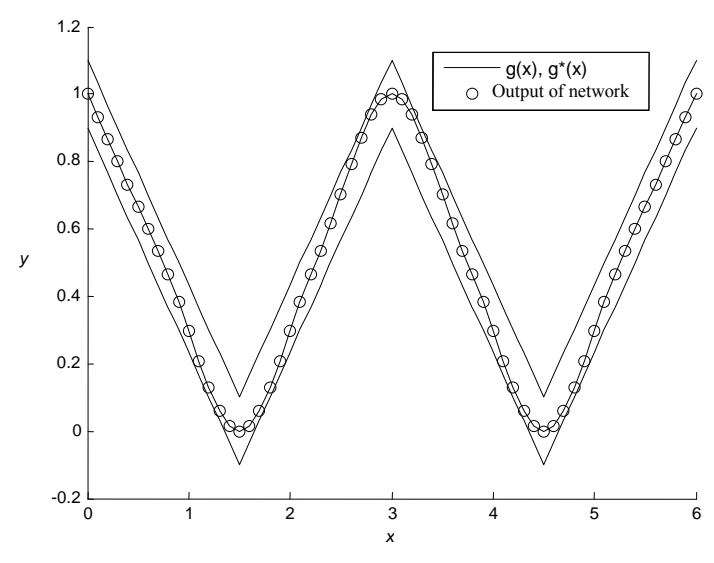

Fig. 5. $r_{i}=3$

\section{CONCLUSIONS}

The neural network using cubic rational spline weight function with quadratic denominator is proposed in this paper, some parameters can be used to control shape, which is much different from the known training process of neural networks.

The new method using rational spline weight functions may be useful to control the position and shape of a curve or surface, which may pay important rules in some applications.

We believe that the method proposed in this paper may be a powerful tool for designing the outer shape of a curve or surface.

\section{ACKNOWLEDGMENT}

I thank P. Han, my graduate student, for his simulation examples given in this paper.

\section{REFERENCES}

[1] D. Y. Zhang, New Theories and Methods on Neural Networks, Beijing: Tsinghua University Press, 2006 (in Chinese).

[2] D. Y. Zhang, "New algorithm for training feedforward neural networks with cubic spline weight functions", Systems Engineering and Electronics, Vol. 28, Sep. 2006, pp.1434-1437 (in Chinese).

[3] D. Y. Zhang, "New algorithm for training neural networks based on generalized Чебышев polynomials", Systems Engineering and Electronics, Vol. 30, Nov. 2008, pp. 2274-2279 (in Chinese).

[4] D. Y. Zhang, "A new algorithm of neural networks with B-Spline weight functions", Proc. 2010 International Conference on Artifical Intelligence and Education. Hangzhou: IEEE Press, 2010, pp.782785.

[5] R. J. Ning, G. Q. Zhu, "The construction of a kind of rational spline", Numerical Mathematics: A Journal of Chinese Universities, Vol. 2,1996, pp. 134-138.

[6] F. N. Fritsch, R. E. Carlson. "Monotone piecewise cubic interpolation", SIAM Journal on Numerical Analysis, Vol. 17, Feb. 1980, pp. 238-246. 